\title{
Un modelo de bajo crecimiento: la informalidad como restricción estructural
}

\author{
Mario Cimoli, Annalisa Primi y Maurizio Pugno
}

Mario Cimoli

Oficial Económico

CEPAL

•rmario.cimoli@cepal.org

Annalisa Primi

Experta en desarrollo tecnológico CEPAL

• annalisa.primi.@cepal.org

Maurizio Pugno

Profesor,

Universidad de Trento, Italia

• maurizio.pugno@economia.unitn.it

$\mathrm{T}$

ras años de reformas y debates sin fin, sigue sin respuesta la pregunta de por qué América Latina no crece más. El presente artículo aborda el tema desde una perspectiva no convencional. Se presenta la persistencia de la informalidad como una barrera estructural para el crecimiento. Como esquema analítico de referencia, introduce un modelo de crecimiento $2 \times 2$, en que solo dos sectores, el formal y el informal, constituyen la economía. El modelo presenta los vínculos entre el patrón de crecimiento del sector formal y las dinámicas del sector informal, y entre estas y el patrón de crecimiento de la economía agregada. Los patrones de especialización adversos y una inserción desfavorable en el comercio internacional alimentan la informalidad. De ahí que el crecimiento liderado por las exportaciones se asemeje más a un modelo de enclave que ni siquiera garantiza un crecimiento elevado, ya que la dinámica propia del sector informal, que concentra alrededor de la mitad de la fuerza de trabajo urbana, afecta negativamente el desempeño de toda la economía. 


\section{Introducción}

Después de años de reformas e interminables discusiones aún no está claro por qué América Latina no crece más. Acelerar el incremento de la productividad, la producción y el empleo es hoy, más que nunca antes, un triple desafío para la región. Si bien es cierto que las reformas estructurales del decenio de 1990 proporcionaron estabilidad macroeconómica y permitieron poner coto a las presiones inflacionarias, hay creciente consenso en que se precisan medidas adicionales para lograr un crecimiento sostenido del producto y reducir la pobreza. En realidad, crecimiento y pobreza siguen siendo asuntos cruciales para la región. En una perspectiva de largo plazo, la comparación del desempeño actual de la región con el del período anterior a la "década perdida" no es alentadora. En el período 1945-1980, la tasa de crecimiento del PIB latinoamericano fue en promedio de $5,6 \%$, mientras que solo alcanzó a 3,8\% entre 1990 y 1997 y a 2,5\% entre 1998 y 2004, registrando una recuperación únicamente en los últimos años. Por su parte, la tasa anual media de crecimiento del ingreso per cápita fue de 3,1\% entre 1945 y 1980 , de $1,9 \%$ en los siete años que siguieron a la década perdida y de $0,5 \%$ entre 1998 y 2004 . Al mismo tiempo, el problema de la pobreza subsiste aún: entre 1945 y 1980 el promedio de hogares pobres fue de 35\%, en el período 1990-1997 de 35,5\% y en 2002 de 36,1\% (CEPAL, 2002, 2004a y 2005; Cimoli, Primi y Stumpo, 2004). Pese a la ola de optimismo alentada por la reciente aceleración del ritmo de crecimiento, la convergencia del producto interno bruto (PIB) por persona de América Latina con el de aquel en la frontera tecnológica es aún una meta por alcanzar. Al tratar de comprender las razones de este fenómeno, el presente trabajo adopta una perspectiva no convencional, es decir, se centra en la presencia de un sector informal considerado como una barrera estructural para

\footnotetext{
$\square$ Una versión anterior del presente trabajo fue presentada en el taller GRADE "A Micro Approach to Poverty Analysis", organizado por la Universidad de Trento, Italia, en febrero de 2005, y también en la reunión del grupo de trabajo sobre política industrial de la Iniciativa para el Diálogo sobre Políticas (IPD), realizada en Río de Janeiro en marzo de 2005. Los autores agradecen especialmente los valiosos comentarios de Oscar Altimir, Giovanni Dosi y Richard Nelson.
}

el crecimiento sostenido. Por ende, la existencia simultánea del sector formal y del informal aparece como una forma peculiar de heterogeneidad estructural que obstaculiza el desarrollo de la región.

Como marco de referencia para el análisis, junto con presentar alguna evidencia empírica, se formula un modelo 2 x 2 que supone la existencia de solo dos sectores en la economía: el formal y el informal. ${ }^{1}$ Este modelo, siguiendo un enfoque estructuralista, examina la relación entre el patrón de crecimiento del sector formal y las dinámicas del sector informal, así como los efectos de esas dinámicas sobre el patrón de crecimiento agregado, y cumple con el propósito de relacionarlas, de manera muy estilizada, en lo que constituye un marco de crecimiento lento. La presencia de patrones de especialización desfavorables y una participación de "baja calidad" en el comercio internacional alimentan dinámicas también adversas en el sector formal, contribuyendo así a la expansión del sector informal. De ahí que se argumente que el patrón de crecimiento exportador de América Latina es más bien de enclave y que ni siquiera garantiza elevadas tasas de crecimiento, ya que la concentración de alrededor de la mitad de la fuerza de trabajo urbana en el sector informal reduce el desempeño de la economía en su conjunto.

\footnotetext{
${ }^{1}$ El supuesto de que en la economía hay solo dos sectores es una simplificación, ya que en realidad los hechos son mucho más complejos y existen diferentes estratos productivos que de varios modos desdibujan tal demarcación. Sin embargo, los modelos 2 × 2 son un instrumento corriente en la literatura económica, especialmente en casos de heterogeneidad estructural, en los que se identifica un sector orientado a los bienes comerciables y otro a los no comerciables. El modelo presentado en este artículo puede ser un caso particular de esa índole. En nuestro caso, como es obvio, el supuesto de que la economía se compone solo de un sector formal y de otro informal se adopta exclusivamente para simplificar el modelo. Al mismo tiempo, ese supuesto encuentra respaldo en estimaciones empíricas relativas a la persistencia de la cuota de empleo urbano que se concentra en el sector informal, que alcanza a alrededor del $50 \%$ del total. Asimismo, cabe señalar que existe una amplia literatura en torno a la heterogeneidad estructural del sector formal, especialmente en relación con la industria manufacturera (Pinto, 1970 y 1976; Cimoli, 2005), y que es posible utilizar un marco analítico similar al propuesto en este artículo para matizar la heterogeneidad estructural en el sector formal, aplicando a los estratos de baja productividad del sector formal las dinámicas previstas aquí para el sector informal.
} 
En consecuencia, se aboga por un cambio en el patrón de especialización del sector formal para modificar la especialización desfavorable del comercio internacional y para acelerar a la vez el dinamismo de la productividad y de la producción en el sector formal, con miras a acrecentar el empleo formal, reducir el peso del sector informal y mejorar el desempeño de la economía agregada.

Antes de introducir el modelo se precisa alguna aclaración sobre el concepto de informalidad. En realidad, el concepto de economía informal data de una misión de la Organización Internacional del Trabajo (оIт) a Kenya en 1972; en esa ocasión se le utilizó por primera vez para identificar a la enorme masa de trabajadores pobres que sobrevivían, producían y realizaban actividades de subsistencia fuera del marco legal, trabajando en el que entonces se denominaba "sector no estructurado" (OIT, 1972).

En los estudios sobre el tema del empleo informal, hoy es posible identificar al menos tres corrientes principales, que difieren en sus definiciones y propuestas de política (Rosenbluth, 1994; Maldonado, 1995). De acuerdo con el enfoque ortodoxo, que es con mucho el que predomina, el sector informal es consecuencia de la intervención estatal: el exceso de reglamentación y requisitos burocráticos hacen que la participación en el sector formal sea una opción extremadamente onerosa (De Soto, 1986; Loayaza, 1997). Por lo tanto, más que un problema en sí, el empleo informal aparece como una solución voluntaria para actores económicos racionales que optan por él debido a las excesivas reglamentaciones y obligaciones que impone el sector formal. De ahí que la flexibilización del mercado de trabajo, la definición de los derechos de propiedad y la reducción de la reglamentación estatal garantizarían una merma del sector informal. Paradójicamente, se supone que tal legalización puede suplir la capacidad empresarial y generar empleo. Un criterio aun más optimista sostiene que al trasladarse al sector informal los trabajadores ganan en flexibilidad y autonomía de horario de trabajo, de modo que no es necesario aplicar políticas de bienestar. ${ }^{2}$ No hay duda de que el enfoque ortodoxo capta algunos aspectos de las dinámicas del sector informal, pero no da cuenta de la profunda precariedad y marginación que caracterizan a la mayor parte de las actividades

\footnotetext{
2 Maloney (2004) ofrece pruebas al respecto. Por el contrario, Goldberg y Pavcnik (2003) ofrecen evidencia empírica sobre las desventajas del empleo informal con respecto al formal.
}

informales en los países en desarrollo y, al no considerar las relaciones entre el dinamismo del sector formal y el empleo informal, no logra explicar por qué América Latina no crece más.

Una visión levemente distinta del sector informal es la que ofrece el enfoque institucional (Piore y Sabel, 1984). La globalización y la creciente interdependencia de las tendencias mundiales contribuyeron a aumentar la inestabilidad de la demanda y a generar cambios radicales en los métodos de producción y en la organización del trabajo. El deseo de maximizar las utilidades llevó a las empresas a adoptar estrategias de reducción de costos basadas en nuevas modalidades contractuales, como la contratación externa y la subcontratación, dando lugar así a una especie de trabajo "informal" o a una "informalización de las actividades formales". ${ }^{3}$ Esta visión, sin embargo, se aplica más a agentes económicos de países desarrollados que trasladan su actividad productiva a otros países para aprovechar diferencias de salarios, que a lo que sucede en las economías en desarrollo.

Un tercer enfoque es el estructuralista, cuyos precursores fueron Prebisch, Pinto y los integrantes del Programa Regional del Empleo para América Latina y el Caribe (PREALC). Según ellos, el sector informal tiene su origen en la especialización del comercio internacional y en el consiguiente funcionamiento imperfecto del sector formal, que es incapaz de emplear y capacitar adecuadamente a la mano de obra. El sector informal constituye una suerte de refugio o estrategia de subsistencia para los grupos marginados, y contribuye a reforzar, si no a generar, la exclusión y las tensiones sociales. Así, desde el punto de vista macroeconómico, el sector informal dificulta el crecimiento económico y aumenta las disparidades de salarios. Por lo tanto, los estructuralistas, como alternativa a las políticas de flexibilización y legalización ortodoxas, son partidarios de aplicar políticas industriales y de producción ajustadas a la realidad, que fomenten el cambio estructural y la absorción de mano de obra por el sector formal para acelerar el crecimiento de la economía.

Más allá de las diferencias entre las distintas corrientes de pensamiento, hay consenso en que el sector informal incluye actividades heterogéneas que van

\footnotetext{
${ }^{3}$ Sin embargo, estudios recientes revelan que las grandes empresas han disminuido la subcontratación de microempresas informales (Sánchez, Joo y Zappala, 2001).
} 
desde trabajos ambulantes de carácter precario hasta actividades por cuenta propia y trabajo asalariado. Sin embargo, una característica común e indiscutible de todas las actividades informales es la baja productivi- dad, por el uso de tecnologías obsoletas y trabajo no calificado y por el tamaño reducido de las empresas (Castells, Portes y Benton, 1989; Rosenbluth, 1994; Tokman, 2001; Maloney, 2004).

\section{II \\ El sector informal: una mirada peculiar a la heterogeneidad estructural}

La heterogeneidad estructural no es un rasgo nuevo del sistema productivo de América Latina. Ya Prebisch (1949, 1962 y 1970), ${ }^{4}$ Singer (1950) y Pinto (1970 y 1976) habían observado el dualismo que se daba en la región y habían subrayado que existían al mismo tiempo un sector orientado principalmente al exterior y altamente productivo y un conjunto de actividades de baja productividad orientadas al mercado interno. La reasignación de los factores productivos de las actividades de baja productividad a las de alta productividad se consideraba como una condición necesaria para que la economía se moviera en un sendero de desarrollo virtuoso. El proceso de convergencia de las actividades de baja productividad con las de alta productividad implicaría un cambio estructural, representado por el aumento del peso de la manufactura en el total de la economía. La industria se veía como el vector principal del crecimiento de la productividad. Según este enfoque, el desarrollo industrial generaría los encadenamientos hacia delante y hacia atrás, los efectos de derrame, la acumulación de capital y las externalidades tecnológicas que se necesitarían para sostener rentabilidades crecientes. La condición periférica se superaría gracias a la reducción de la heterogeneidad estructural, identificada como la concentración del empleo en actividades de baja productividad.

Hoy en día, en economías abiertas y tras una década de reformas, la heterogeneidad sigue siendo una debilidad estructural del sistema productivo latinoamericano, y la concentración del progreso técnico no sólo persiste sino que se ha acentuado. La apertura ha favorecido una reorientación de los patrones de

\footnotetext{
${ }^{4}$ El ensayo de Prebisch (1949) apareció posteriormente en Prebisch
} (1962). Se citan ambos por ser el segundo más fácil de encontrar. especialización hacia actividades que hacen uso intensivo de recursos naturales y mano de obra, junto con la privatización, la modernización y el avance tecnológico en la producción. Estos procesos han transformado las dinámicas del sector formal, afectando adversamente las capacidades tecnológicas endógenas, reduciendo los encadenamientos internos y la capacidad de absorción de fuerza de trabajo del sector formal manufacturero y disminuyendo así la capacidad del mismo sector de actuar como vector del desarrollo de toda la economía (Cimoli, 2005). La progresiva erosión de la capacidad de absorber empleo que ha mostrado el sector formal manufacturero ha aumentado el desempleo y ha nutrido al sector informal urbano, que ha ido absorbiendo la fuerza de trabajo residual. Por ende, la existencia simultánea de un sector moderno orientado al exterior que no provee suficiente empleo constantemente y de un sector informal de baja productividad donde el empleo se concentra, aparece como un rasgo peculiar de la heterogeneidad estructural. De ahí que América Latina se asemeje a una economía polarizada, en la cual coexisten dos sectores con dinámicas distintas de acumulación, producción y crecimiento y el sector formal aparece como un enclave que lidera el crecimiento.

Las estadísticas sobre el empleo informal no son alentadoras. ${ }^{5}$ Se estima que en América Latina, en el año 2003, el 47\% del empleo urbano era informal (CEPAL, 2005; OIT, 2004; Weller, 2000). El peso del

\footnotetext{
${ }^{5}$ El sector informal es por definición difícil de identificar y de medir. Según el enfoque estándar de las Naciones Unidas (al que se ajustan las metodologías de la CEPAL y de la OIT), el sector informal se compone de actividades de baja productividad e incluye las siguientes categorías: servicio doméstico, trabajadores asalariados no profesionales, trabajo familiar no remunerado, y personas empleadas en microempresas con menos de cinco trabajadores.
} 


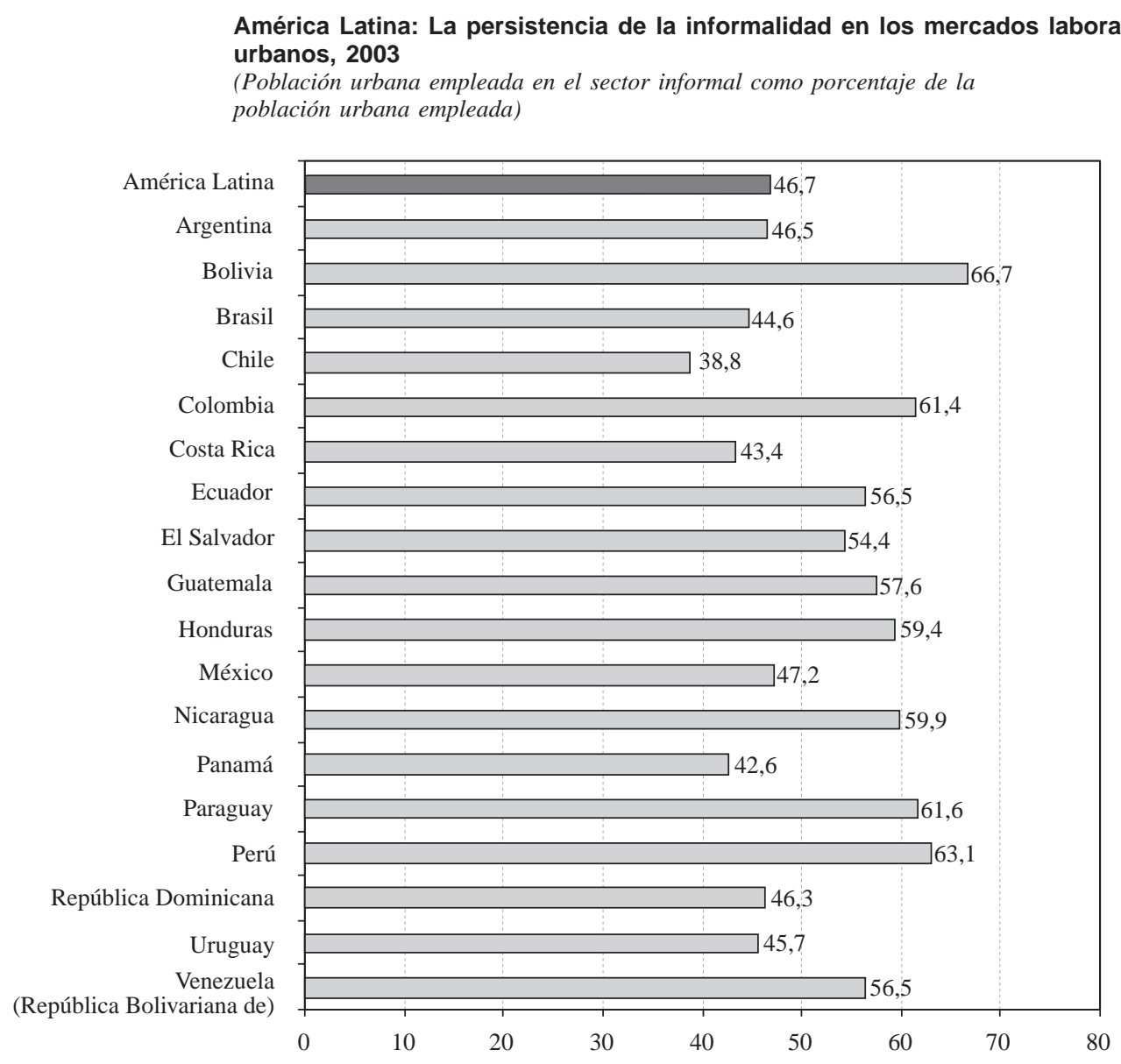

Fuente: Elaboración propia con datos de la CEPAL y la OIT. Las cifras son estimaciones basadas en encuestas nacionales de hogares y se refieren a la población urbana empleada en sectores de baja productividad del mercado de trabajo, que se consideran como una variable sustitutiva del sector informal.

sector informal en los mercados laborales urbanos es bastante homogéneo entre los países de la región, yendo del $39 \%$ en Chile al $67 \%$ en Bolivia. En realidad, pese a las diferencias entre los países de la región relativas a los patrones de especialización y a las divergencias en sus estructuras exportadoras, ${ }^{6}$ la persistencia del empleo informal es una característica común de las economías latinoamericanas (gráfico 1).

Los análisis empíricos del sector informal encuentran serias limitaciones debido a la naturaleza misma

\footnotetext{
${ }^{6}$ En la región coexisten patrones de especialización y comercialización divergentes: algunos hacen uso intensivo de mano de obra, otros hacen uso intensivo de recursos naturales y otros se centran básicamente en actividades primarias o terciarias. Véase en Cimoli (2005) un estudio actualizado sobre la heterogeneidad estructural en América Latina.
}

de este fenómeno, difícil de identificar y medir. En el cuadro 1 se incluye una somera cuantificación de las diferencias estructurales en América Latina, considerando la distribución del empleo y la productividad del trabajo en el sector formal y el informal, en comparación con las del total de la economía. ${ }^{7}$ En 1990 el 44\% del empleo urbano era informal, mientras que en el 2000 lo era el $45 \%$.

\footnotetext{
${ }^{7}$ Las estimaciones de la distribución del empleo y de la productividad del trabajo se basan en datos de la CEPAL derivados de las encuestas nacionales de hogares. La productividad del trabajo en el sector informal se estima a partir de los ingresos medios de los trabajadores informales; tales ingresos se consideran una buena variable sustitutiva del valor agregado generado por el sector informal, debido a la naturaleza de las actividades realizadas en este último.
} 
Cada vez más la informalidad es un rasgo persistente de las economías latinoamericanas. No solo tiene efectos adversos en el desempeño de la economía en términos de exclusión, marginalización, precariedad y disparidades de salarios, sino que afecta directamente la productividad agregada y por ende el crecimiento, reduciendo la productividad media de la economía. Las actividades informales son de baja productividad y la concentración de la fuerza de trabajo en el sector menos productivo hace caer la productividad agregada. Se estima que a fines del decenio de 1990 la productividad del trabajo en el sector informal era solo el $20 \%$ de aquella en el sector formal, y que el sector informal concentraba alrededor de la mitad del empleo total (cuadro 1).

En 1990 casi la mitad de la fuerza de trabajo se concentraba en un sector cuya productividad representaba un $48 \%$ de la productividad agregada de la economía y alrededor de $33 \%$ de la productividad del sector formal. A fines de la década la situación era aún más preocupante. En el 2000, alrededor de la mitad del empleo urbano se hallaba en al sector informal, cuya productividad representaba solo el $30 \%$ de la productividad agregada y el $20 \%$ de la productividad del sector formal. En el cuadro 2 se aprecia la brecha cada vez mayor entre la productividad del sector formal y la del informal en cuatro países de la región.

El hecho de que en promedio alrededor de 50\% del empleo se concentre en el sector informal debe tener considerables consecuencias sobre el crecimiento agregado. El análisis de las dinámicas de dicho sector puede ayudar a entender por qué América Latina no crece más, o no todo lo que debiera. Como marco analítico se introduce un modelo simple de crecimiento ajustado a una economía dual peculiar, en la que coexiste un sector formal con otro informal.

El modelo se focaliza en la disparidad entre el sector formal y el informal en función del crecimiento, la productividad, la distribución del empleo, y los salarios, por razones de simplificación. Sin embargo, es necesario destacar que cada uno de estos sectores dista de ser homogéneo. El sector formal comprende actividades bastante diversas — como industrias que

CUADRO 1

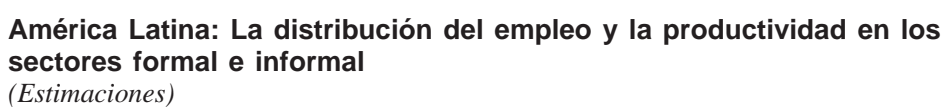

\begin{tabular}{|c|c|c|}
\hline \multirow[t]{2}{*}{ Año } & Sector formal & Sector informal \\
\hline & \multicolumn{2}{|c|}{ Distribución del empleo (\% de trabajadores) } \\
\hline 1990 & 55,6 & 44,4 \\
\hline \multirow[t]{2}{*}{2000} & 54,6 & 45,4 \\
\hline & \multicolumn{2}{|c|}{ Productividad del trabajo (productividad agregada $=100$ ) } \\
\hline 1990 & 141,8 & 47,6 \\
\hline 2000 & 157,8 & 30,5 \\
\hline
\end{tabular}

Fuente: Cimoli, Correa y Primi (2003).

CUADRO 2

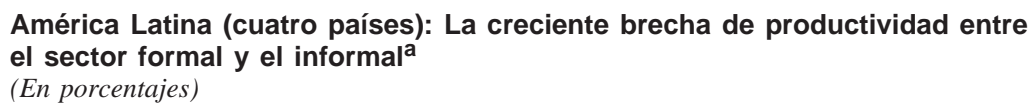

\begin{tabular}{llr}
\hline Países & \multicolumn{2}{c}{ Productividad del sector informal/productividad del sector formal } \\
\cline { 2 - 3 } & 1990 & 2000 \\
\hline Brasil & 40,3 & 20,1 \\
México & 31,9 & 28,2 \\
Uruguay & 24,2 & 16,9 \\
Venezuela & & 32,9 \\
(República Bolivariana de) & 39,5 & 32,9 \\
\hline
\end{tabular}

Fuente: Estimaciones propias con datos de la CEPAL.

a La productividad relativa es la productividad del trabajo en el sector informal y en el sector formal. 
hacen uso intensivo de mano de obra o de conocimiento y otras basadas en el procesamiento de recursos naturales_-, las que exhiben dinámicas distintas en materia de producción, productividad y empleo (Cimoli, 2005). Sin embargo, esas actividades tienen en general características comunes, como la orientación hacia el sector externo, la relativa modernización de los procesos productivos, la mano de obra relativamente calificada y el tamaño mediano y grande de las empresas. Por su parte, el sector informal abarca actividades heterogéneas (de hecho, incluye actividades empresariales y trabajo asalariado), pero es más homogéneo en cuanto a su baja productividad, los estándares de vida de los trabajadores y la poca calificación de la mano de obra. ${ }^{8}$

El análisis se desarrolla a partir de un tradicional modelo $2 \times 2$. En particular, se supone una economía dual, constituida por un sector formal y otro informal. La producción del sector formal, $Y_{f}$, utiliza insumos importados y se exporta, mientras que la del sector informal, $Y_{i}$, se destina exclusivamente al mercado interno. ${ }^{9}$

Según el enfoque de los salarios de eficiencia, que garantiza la segmentación en el mercado del trabajo, la productividad y los salarios son mayores en el sector formal que en el informal. ${ }^{10}$ Basándose en dicho enfoque, las empresas del sector formal fijan un margen por encima de los salarios flexibles del sector informal, considerando la productividad del sector formal. ${ }^{11}$

En ese contexto, los trabajadores se disputan los empleos formales, y las empresas del sector formal pueden seleccionar el factor trabajo, lo que contribuye a que su productividad sea mayor. En el sector formal, una demanda dada determina las importaciones y las necesidades de fuerza de trabajo, mientras que el sector informal simplemente absorbe la fuerza de trabajo residual. El carácter residual del empleo informal permite mantener la segmentación del mercado del trabajo y fortalece la restricción externa al crecimiento.

\section{III}

\section{Las dinámicas del producto, de la productividad y del empleo: crecimiento en una economía dual}

Las principales ecuaciones del modelo que expresan las dinámicas en el sector formal son la de régimen de demanda y la de régimen de productividad (Setterfield y Cornwall, 2002). La primera explica el crecimiento de la demanda en una economía abierta, y la segunda

\footnotetext{
${ }^{8}$ El concepto de informalidad debe distinguirse del de ilegalidad, aunque en ciertos casos los dos puedan coincidir. Las actividades informales difieren de las ilegales porque conciernen productos o servicios legales desarrollados de forma ilegal. Además, el análisis del sector informal en los países en desarrollo debe realizarse con una perspectiva diferente de la que se adopta en los países desarrollados, donde la informalidad más bien se asocia con la evasión fiscal y el aporte en forma ilegal de insumos para el sector formal. ${ }^{9}$ El apéndice A explica la parte estática del modelo y da más detalles analíticos sobre las especificaciones utilizadas.

${ }^{10}$ En la teoría de los salarios de eficiencia la productividad del trabajo está positivamente relacionada con los salarios, y la brecha salarial entre industrias o sectores se debe a diversos factores (entre otros, incentivar el esfuerzo del trabajador aumentando el costo oportunidad de perder el empleo, y elevar el nivel del capital humano atrayendo a los trabajadores con salarios de reserva más altos). Existen numerosos estudios basados en la teoría de los salarios de
}

internaliza el crecimiento de la productividad de manera circular con el crecimiento de la demanda.

El régimen de demanda representa el crecimiento de la producción del sector formal, $y_{f}$, debido a la demanda de exportaciones. ${ }^{12}$ Las especificaciones eficiencia en economías duales: Stiglitz (1974 y 1976), Bulow y Summers (1986), Esfahani y Salehi-Isfahani (1989), Agenor y Aizenman (1999), Goldberg y Pavcnik (2003). Arbache (2001) proporciona alguna información sobre Brasil. Los cuadros 1 y 2 muestran alguna evidencia empírica sobre la brecha de productividad entre el sector formal y el informal, mientras que, más adelante, el gráfico 4 muestra la brecha de salarios entre los dos sectores.

${ }^{11}$ La brecha de salarios entre los dos sectores es una consecuencia del enfoque de los salarios de eficiencia. Además, el margen que se impone sobre los salarios del sector informal y que determina los salarios en el sector formal depende, también, de que el factor trabajo no sea sustituible y de los arreglos institucionales del mercado del trabajo formal que pueden afectar a los salarios del mismo sector. ${ }^{12}$ La inclusión del componente de crecimiento de la demanda interna no cambiaría los resultados del modelo, aunque el crecimiento se redujera, como es el caso en América Latina. 
siguen la perspectiva de Harrod-Kaldor, ${ }^{13}$ como lo describe sintéticamente la ecuación (9). ${ }^{14}$

$$
y_{f}=b \pi_{f} \text { Régimen de demanda (RD) }
$$

$$
b=\frac{x}{\varepsilon \pi *}
$$

en que $\pi_{f}$ corresponde a la tasa de crecimiento de la productividad del sector formal, $\pi^{*}$ a la tasa de crecimiento de la productividad externa, $x$ indica el incremento de las exportaciones derivado del aumento del ingreso externo y $\varepsilon$ muestra la elasticidad ingreso de las importaciones.

El significado económico del régimen de demanda es simple. ${ }^{15}$ Mientras menor sea la restricción externa, debido al crecimiento de las exportaciones y a la elasticidad-ingreso de las importaciones, mayor será a largo plazo el crecimiento del producto del sector formal, orientado a los mercados internacionales. En especial, la tasa de crecimiento del producto será mayor mientras más rápidamente se supere la brecha de productividad con respecto a la frontera tecnológica, es decir, $\pi_{f} / \pi^{*}$. En rigor, el aumento de esta relación entraña un incremento de la competitividad en los mercados internacionales. Por su parte, la brecha tecnológica se debe principalmente a la capacidad de aprendizaje tecnológico, a los encadenamientos y a la diversificación de la estructura productiva. ${ }^{16}$ Por lo tanto, el régimen de demanda permite que el modelo capte la capacidad del sector exportador de impulsar el crecimiento económico, generando más competitividad y limitando la restricción externa de acuerdo con las características de la estructura productiva.

13 Véase, entre otros, Cimoli, Dosi y Soete (1986); Cimoli (1988 y 1994); Dutt (2001); Harrod (1933); Kaldor (1966 y 1975); Lawson, Palma y Sender (1989); McCombie y Thirlwall (1994); Thirlwall, 1979.

${ }^{14}$ Esta especificación no tiene en cuenta los efectos del tipo de cambio real, como en el modelo original de Thirlwall que Gonzaga (2003), Moreno-Brid (1999a y 1999b) y Perraton (2003) han puesto a prueba con éxito en varios países de América Latina. El modelo propuesto por Frenkel y Taylor (2006) en su reciente trabajo sobre la relación entre el tipo de cambio real y el desarrollo, y en particular sobre los efectos del tipo de cambio real en el mercado del trabajo de una economía con dos sectores, uno especializado en bienes comerciables y otro en no comerciables, ofrece consideraciones interesantes al respecto.

${ }^{15}$ En el apéndice B se presentan las derivaciones económicas y se ofrecen más detalles al respecto.

16 Véase en Cimoli y Correa (2005) un análisis empírico detallado de la brecha tecnológica en América Latina, como multiplicador, antes y después del proceso de liberalización.
La ecuación del régimen de productividad sigue la ley de Verdoorn-Kaldor, ${ }^{17}$ es decir,

$$
\pi_{f}=\alpha+\beta y_{f} \text { Régimen de productividad (RP) }
$$

en que $\alpha>0$ y $0<\beta<1$. El parámetro $\alpha$ mide los esfuerzos autónomos que influyen directa e indirectamente sobre el crecimiento de la productividad del sector formal, como son la formación de capital humano y las actividades de capacitación. El coeficiente $\beta$ capta la capacidad estructural de aprendizaje y de difusión del conocimiento, los encadenamientos y las complementariedades de la industria, explicando así que el crecimiento del producto pueda aumentar la productividad. ${ }^{18}$

El régimen de demanda (ecuación 9) y el régimen de productividad (ecuación 11) forman un sistema de ecuaciones simultáneas. Por lo tanto, las soluciones de equilibrio (señaladas por el subíndice $e$ ) correspondientes al incremento del producto y de la productividad en el sector formal son las siguientes:

$$
\begin{aligned}
& y_{f \mathrm{e}}=\frac{b \alpha}{1-b \beta} \\
& \pi_{f \mathrm{e}}=\frac{\alpha}{1-b \beta}
\end{aligned}
$$

Las soluciones de equilibrio del producto y de la productividad en el sector formal dependen de la segmentación del mercado del trabajo y de que la oferta total de trabajo no se vea restringida en la práctica. ${ }^{19}$

Para representar el régimen de demanda y el de productividad en el espacio $\left(\pi_{f}, y_{f}\right)$, se transforma la ecuación (11) en la (14):

\footnotetext{
17 Para mayores detalles sobre la ecuación de Verdoorn-Kaldor, véase Amable (1992); Kaldor (1975); McCombie y Thirlwall (1994); McCombie, Pugno y Soro (2002); Thirlwall (1979 y 1997).

${ }^{18} \mathrm{El}$ aprendizaje se relaciona con la capacidad de los agentes económicos de transformar y mejorar la producción industrial y las técnicas de organización, mientras que los encadenamientos y la densidad de las redes determinan la posibilidad de difundir tecnologías y conocimientos (Dosi y Freeman, 1992; Dosi, Pavitt y Soete, 1990).

${ }^{19}$ Estas condiciones en general corresponden a algunos de los supuestos en la literatura sobre la restricción externa al crecimiento (McCombie y Thirlwall, 1994); sin embargo, en este modelo los salarios no se determinan exclusivamente en el sector formal, debido al enfoque de los salarios de eficiencia. Pugno (1998) presenta un modelo de crecimiento que analiza a la vez la restricción externa y la restricción de la oferta de trabajo al crecimiento.
} 


$$
y_{f}=-\frac{\alpha}{\beta}+\frac{1}{\beta} \pi_{f}
$$

y por ende la (9) y la (14) pueden representarse en el gráfico 2.

Para garantizar soluciones positivas es necesario suponer que $b \beta<1$, que es una hipótesis realista. El patrón de crecimiento del producto y de la productividad es mejor cuanto mayores sean los encadenamientos y las capacidades internas de transferir capacidades; es decir, cuanto más compleja sea la estructura productiva, más intensos los esfuerzos de formación de capital humano y las actividades de capacitación, y cuanto más virtuosa sea la participación en el comercio internacional: en otras palabras, cuanto menor sea la elasticidad-ingreso de las importaciones respecto del crecimiento de las exportaciones.

La misma condición que garantiza que las soluciones de equilibrio sean positivas, implica que el equilibrio sea estable desde el punto de vista dinámico. ${ }^{20}$

\section{GRÁFICO 2}

Situación de equilibrio estable: crecimiento del producto y de la productividad en el sector formal

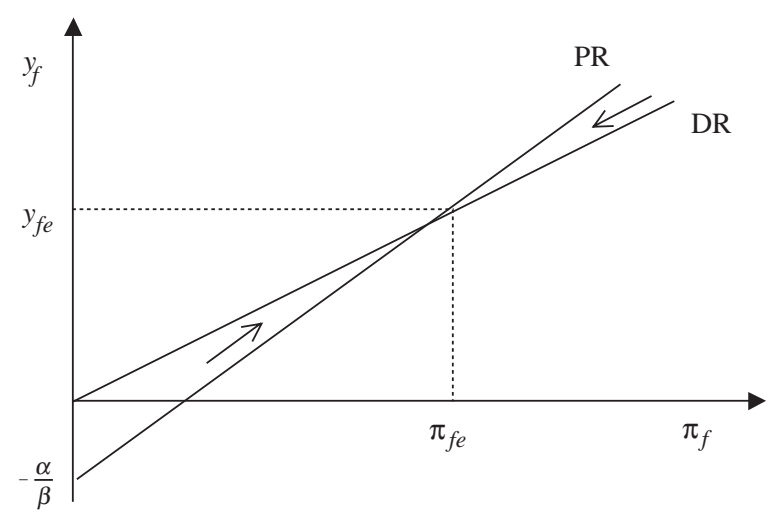

Fuente: Elaboración propia.

\footnotetext{
${ }^{20} \mathrm{Si}$ el valor inicial de $\pi_{f}$ y de $y_{f}$ se encuentra fuera del punto de equilibrio, posiblemente tras un cambio de los parámetros de medición, se produce un proceso circular entre el régimen de demanda y el régimen de productividad del sector formal, y el crecimiento de la productividad y de la producción terminan por converger hacia los valores de equilibrio. Paus, Reinhardt y Robinson (2003) demuestran, a partir de la prueba de causalidad de Granger, que en América Latina hay una relación de causalidad relevante entre el crecimiento de la productividad y el de las exportaciones, y entre este último y el de la productividad.
}

A partir de la definición de productividad, es decir, $\Pi_{f}=\left(Y_{f} / L_{f}\right)$, se obtiene:

$$
\pi_{f}=y_{f}-l_{f}
$$

De las sustituciones correspondientes se deriva la tasa de crecimiento de equilibrio del empleo formal:

$$
l_{f \mathrm{e}}=-\alpha \frac{1-b}{1-b \beta}
$$

La ecuación (16) pone de relieve la importancia de las características de la estructura productiva y del patrón de comercio exterior para la dinámica del empleo. De hecho, la tasa de equilibrio del empleo formal será negativa o positiva según si $(0<b<1)$ o $(1<b<1 / \beta)$, es decir, dependiendo de la elasticidad-ingreso de las importaciones y la tasa de crecimiento de las exportaciones (véase más atrás la ecuación 10).

Cuando $b<1$, un aumento de $\beta$, que capta los encadenamientos industriales, la difusión de conocimientos, la complementariedad y el capital humano, es favorable para el crecimiento de la productividad, pero no implica un incremento del empleo en el sector formal. Esto se debe a la restricción externa que necesariamente obstaculiza el crecimiento de la producción. Si $b>1$, el aumento de $\beta$ afectará positivamente el crecimiento tanto de la productividad como de la producción, ya que la restricción externa no es tan determinante.

De ahí que el crecimiento liderado por las exportaciones pueda determinar una dinámica viciosa debido a las características de la estructura productiva y al patrón de participación en el comercio internacional, que puede reforzar la restricción externa al crecimiento. Al mismo tiempo, el mejoramiento de la productividad en el sector formal puede venir acompañado de una reducción del empleo en este sector, acrecentando con ello el empleo informal y reduciendo aun más el crecimiento de la economía agregada.

El gráfico 3 muestra el dinamismo de la producción, de la productividad y del empleo en el sector formal latinoamericano durante las tres últimas décadas. El patrón de crecimiento suavizado de la productividad del trabajo, tras un período de crecimiento bajo pero estable, se dispara, especialmente a causa de la expulsión de fuerza de trabajo, para luego volver a caer, sin recuperar al mismo tiempo la capacidad de absorción de mano de obra. 
GRÁFICO 3

América Latina: Dinámicas de la producción, la productividad y el empleo en el sector formal
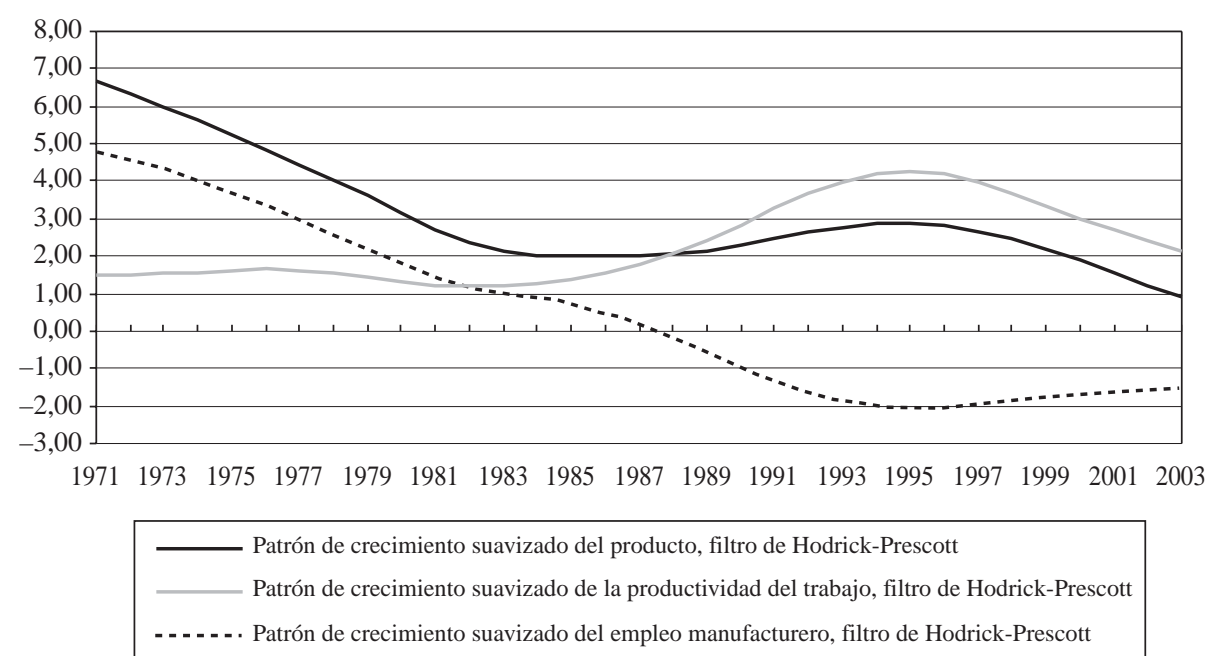

Fuente: Elaboración propia con datos de la CEPAL y del Programa de Análisis de la Dinámica Industrial (PADI) de la CEPAL.

Por otro lado, el patrón monótonamente decreciente de la producción es acompañado por el deterioro del crecimiento del empleo en la industria manufacturera, crecimiento que se vuelve negativo desde fines de la década de 1980. Este es un hecho poco usual en una economía en desarrollo. En la República de Corea, por ejemplo, el incremento de la productividad en las tres últimas décadas marchó parejo con el crecimiento del empleo en la industria manufacturera (Cimoli, Primi y Stumpo, 2004). En los decenios de 1950 y 1960 los países actualmente desarrollados se comportaron de la misma manera (Kaldor, 1966), y solo después, cuando ya habían alcanzado la frontera tecnológica, sufrieron la caída del empleo en el sector manufacturero. Además, ese proceso de "desindustrialización", típico de algunos países europeos que muestran caídas en el empleo manufacturero, es fundamentalmente distinto al de erosión de la capacidad de absorber empleo en las industrias latinoamericanas.

El desarrollo del sector de los servicios ha cambiado la distribución del empleo a nivel mundial. Sin embargo, en los países europeos la reasignación del factor trabajo, que además se ha traducido en un incremento del empleo en las actividades emergentes y más dinámicas, se ha visto acompañada por importantes esquemas de asistencia estatal y políticas de bienestar. América Latina, por el contrario, ha exhibido un patrón diferente, ya que la reasignación del factor trabajo ha dado lugar a un incremento del empleo en los sectores de más baja productividad, sobre todo en las actividades de servicios personales y comerciales (Tokman, 1997; CEPAL, 2005; OIT, 2004).

En América Latina, el deterioro del empleo en el sector formal ha aumentado el desempleo y el empleo informal (Ros, 2005; BID, 2003; Pliego, 1997). Para reflejar este hecho, en el modelo se supone que el sector informal desempeña un papel residual, con lo cual surge un singular modelo de "pleno empleo" donde:

$$
L=L_{f}+L_{i}
$$

de manera que:

$$
l=\lambda l_{i}+(1-\lambda) l_{f} \quad 0 \leq \lambda \leq 1
$$

De acuerdo con la ecuación (18), la tasa de crecimiento de la fuerza de trabajo $l$ se descompone en crecimiento del empleo en el sector formal y crecimiento del empleo en el sector informal; $\lambda$ representa la participación del empleo informal en la economía. Por lo tanto, habiendo determinado la dinámica del empleo en el sector formal, y dado que el crecimiento de la fuerza de trabajo es de carácter exógeno, se obtiene la tasa de equilibrio del empleo en el sector informal: 


$$
l_{i e}=\frac{l}{\lambda}+\alpha \frac{1-\lambda}{\lambda} \frac{1-b}{1-b \beta}
$$

Esta ecuación significa que el empleo informal aumenta cuando $l>(1-\lambda) l_{f}$, es decir, para que $l_{i \mathrm{e}}>0$ no es necesario que $l_{f \mathrm{e}}$ tenga valor negativo.

Es arriesgado formular hipótesis sobre la dinámica de la productividad del sector informal; sin embargo, es razonable estimar que la tasa de crecimiento de la productividad en él depende de la tasa de crecimiento de la productividad en el sector formal. Por lo tanto, se considera que:

$$
\pi_{i}=c \pi_{f} \quad \text { en que } 0 \leq c \leq 1
$$

Por consiguiente:

$$
\pi_{i e}=\frac{c \alpha}{1-b \beta}
$$

En consecuencia, se obtiene la tasa de equilibrio del crecimiento del producto informal a partir de las ecuaciones (19), (20) y de la usual descomposición del crecimiento de la productividad:

$$
y_{i \mathrm{e}}=\frac{l(1-\beta b)+\alpha((1-\lambda)(1-b)+\lambda c)}{\lambda(1-b \beta)}
$$

Un mayor crecimiento de la fuerza de trabajo genera una más alta tasa de crecimiento de la producción informal. Asimismo, una aceleración del crecimiento de la productividad en el sector formal - que se reflejaría en un valor más alto de $\alpha$ - acelera el crecimiento de la producción del sector informal, ya que el excedente de trabajadores del sector formal termina alimentando la actividad informal.

Las dinámicas del sector informal afectan negativamente el desempeño de la economía agregada. Si el valor de $b$ no es lo suficientemente elevado, por ejemplo, si $b<1$, la economía se torna cada vez más informal, tanto en lo referente al empleo como a la producción. La "informalización" del empleo se observa en la ecuación (19), mientras que aquella de la producción se desprende de la ecuación siguiente:

(23) $y_{i \mathrm{e}}-y_{f \mathrm{e}}=\frac{l(1-\beta b)+\alpha(\lambda(1-c)+(1-b))}{\lambda(1-b \beta)}$

que es superior a cero.

El desempeño de la economía también se ve afectado negativamente desde el punto de vista de la equidad. En el caso general en que $c<1$ la brecha salarial entre ambos sectores aumenta, ya que:

$$
w_{f}-w_{i}=\pi_{f} \frac{1-c}{1+d}>0
$$

en que la disminución de $d$ contribuye a ampliar la diferencia de salarios. ${ }^{21}$

El patrón decreciente del índice de ingreso relativo del sector informal con respecto al del sector formal confirma la tendencia a que se agrande la brecha de salarios en los países considerados. En efecto, entre 1990 y 2002 el ingreso de los trabajadores informales de América Latina cayó en forma prácticamente sostenida en relación con el de los trabajadores profesionales y técnicos asalariados del sector privado formal. En 2002, la relación entre el ingreso medio de los trabajadores del sector informal latinoamericano y el de esos asalariados del sector formal fue inferior casi en un tercio a la de 1990 (gráfico 4). ${ }^{22}$ En rigor, aunque la persistencia del empleo informal, la pobreza y la desigualdad sean tres problemas estructurales diferentes de la región, ellos están íntimamente relacionados. En realidad, en el decenio de 1990 casi el 60\% de los pobres de América Latina trabajaba en actividades informales (Tokman, 1994), y de acuerdo con una evaluación empírica del BID, el empleo informal generaba entre $10 \%$ y $25 \%$ de la desigualdad de salarios en el mercado del trabajo.

Según el modelo, el crecimiento de la productividad puede disminuir incluso en el caso extremo de que la productividad de ambos sectores aumente al mismo ritmo (caso en que $c=1$ ). El caso más frecuente, el de que $c<1$, es aun más complejo, ya que se amplía la diferencia tanto en el crecimiento de la producción como en el nivel de productividad ( $d$ disminuye). En el caso extremo, la economía tenderá a estabilizarse de la siguiente manera: si $t \rightarrow \infty$, entonces $l_{i \mathrm{e}} \rightarrow l, \lambda \rightarrow 1, y_{i \mathrm{e}} \rightarrow l+c \pi_{\mathrm{fe}}$, es decir, la economía tiende a ser totalmente informal y la tasa de crecimiento de la productividad disminuye hasta coincidir con la del sector informal.

\footnotetext{
${ }^{21}$ Como se define en la ecuación (7) del apéndice A, $d$ expresa la productividad del sector informal como fracción de aquella del sector formal.

22 Esto significa que el ingreso del sector informal cayó a un ritmo más acelerado que el de los salarios del sector formal, con lo cual aumentó la desigualdad entre ambos.
} 


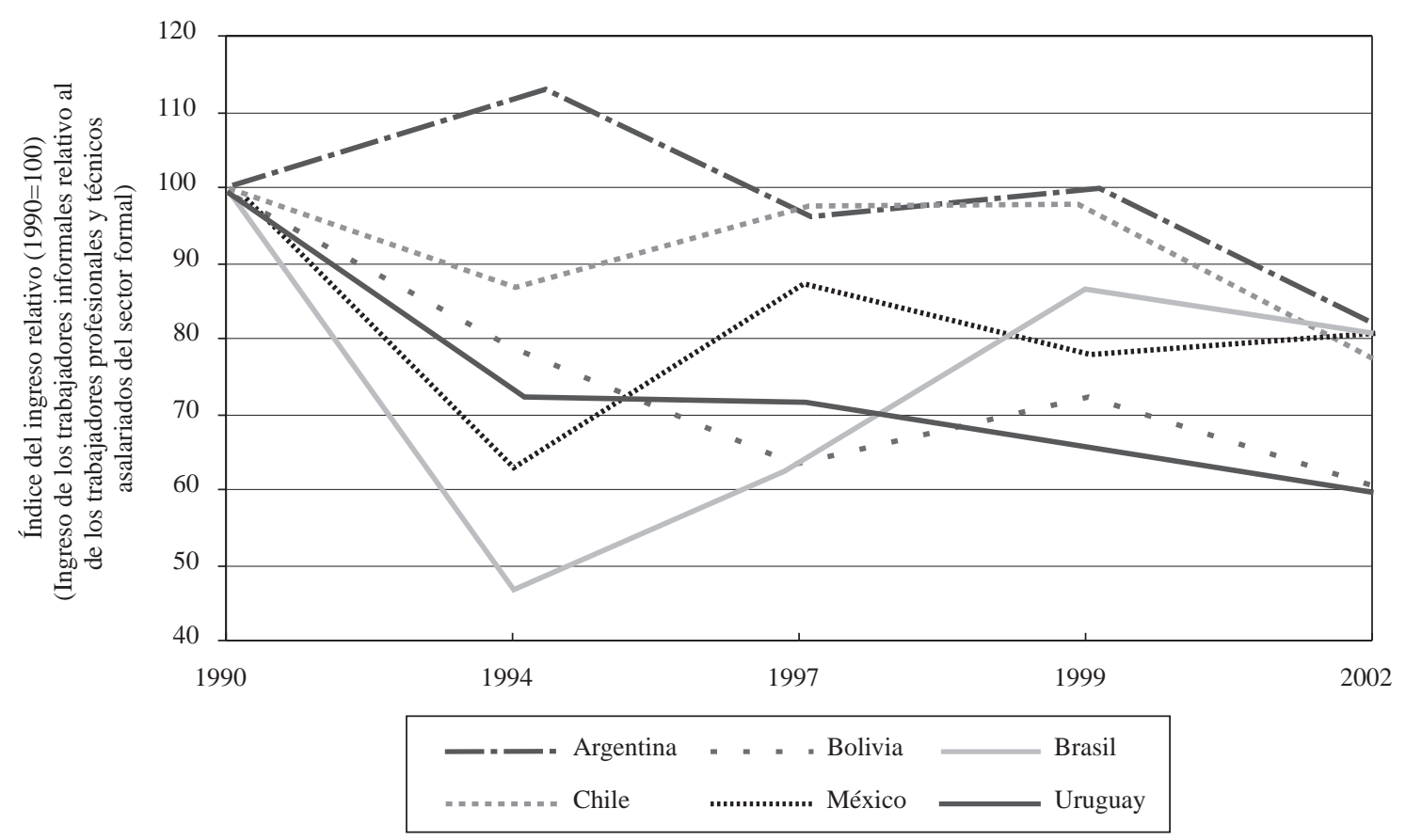

Fuente: Elaboración propia a partir de CEPAL (2003).

a Las cifras corresponden a estimaciones de la CEPAL basadas en encuestas nacionales de hogares. Las series representan el índice (año base 1990) de la relación entre el ingreso medio de los residentes urbanos que trabajan en sectores de baja productividad (es decir, en el sector informal) y el ingreso medio de los profesionales y técnicos asalariados que se desempeñan en el sector privado formal.

Estas conclusiones se contraponen a lo sostenido por la teoría clásica, de acuerdo con la cual el sector informal desaparecería natural y gradualmente con el avance del proceso de desarrollo y modernización. En realidad, de acuerdo con la dinámica del modelo, para generar un dinamismo virtuoso que favorezca al mismo tiempo el incremento del empleo formal y el crecimiento de la producción y la productividad, tendrían que producirse simultáneamente el cambio estructural ${ }^{23}$ y la aplicación cada vez mayor de medidas para estimular los encadenamientos, difundir conocimientos y formar capital humano.

${ }^{23}$ El cambio estructural implica reorientar la especialización del sector formal hacia etapas o industrias con mayor contenido de conocimiento y más diversificadas.
De lo contrario, es posible que persista una modalidad de crecimiento de enclave, en que un sector formal moderno y orientado hacia afuera coexiste con un sector informal creciente, con consecuencias adversas para el crecimiento agregado. Esto es justamente lo que ha estado sucediendo en América Latina: ha habido modernización y ha aumentado el comercio exterior, pero el patrón de especialización y comercialización ha favorecido la expulsión relativa de mano de obra desde el sector formal, alimentando así el crecimiento y la persistencia del sector informal. ${ }^{24}$

${ }^{24}$ Carillo y Pugno (2004) explican la persistencia del empleo informal vinculado con el subdesarrollo y también demuestran que las políticas orientadas a mejorar los resultados del sector formal son más útiles que aquellas destinadas a legalizar las actividades informales. 


\section{IV}

\section{El crecimiento y la informalidad: un ejercicio de estática comparada}

Los regímenes de demanda y de productividad, junto con la relación entre los sectores formal e informal, definen un modelo de crecimiento en el cual las características microeconómicas de la estructura productiva (es decir, la capacidad de generar y transferir conocimientos y tecnología, el patrón de especialización, la participación en el comercio internacional y la coexistencia de un sector formal y de uno informal $)^{25}$ determinan el patrón de crecimiento agregado.

En nuestro marco analítico, las características de la estructura productiva actúan como barreras que limitan el crecimiento liderado por las exportaciones y, en un círculo vicioso, el sector informal contribuye con sus debilidades estructurales a reducir el crecimiento. Por ello se sostiene que el modelo de crecimiento exportador latinoamericano se parece más a un modelo de enclave que ni siquiera garantiza un crecimiento global elevado.

En realidad, el modelo ayuda a esclarecer cuáles son los defectos estructurales que en América Latina obstaculizan el crecimiento impulsado por las exportaciones y señala las barreras que limitan la convergencia de los países de la región con aquellos que se hallan en la frontera tecnológica.

Además de la estabilidad macroeconómica y la formación de capital humano, se necesita un cambio estructural que favorezca la reestructuración del aparato productivo en torno a sectores más complejos y con uso más intensivo de tecnología, para modificar patrones de especialización internacional y acelerar a la vez el crecimiento de la economía.

Prebisch (1949), Nurske (1953) y Kuznets (1980) ya habían sostenido que las estrategias de apertura comercial y de fomento de las exportaciones no implicarían una aceleración del crecimiento. En realidad, es posible que el comercio no sirva para dinamizar el crecimiento cuando el mercado interno no está suficientemente desarrollado. En América

${ }^{25}$ El sector formal y el informal, que operan bajo mecanismos institucionales, organizativos y productivos distintos, determinan a la vez la productividad y el crecimiento agregados.
Latina, la persistencia del empleo informal, la escasa complejidad y articulación de la estructura productiva y los incentivos derivados del abrupto proceso de apertura, han afectado los patrones de especialización y la generación y difusión de capacidades tecnológicas, favoreciendo la permanencia de las actividades informales y desacelerando así el crecimiento. Además, los débiles esfuerzos tecnológicos internos y la falta de capacidades locales para aprovechar los efectos de derrame del cambio técnico hacen que la demanda externa sea un factor determinante del crecimiento de largo plazo de América Latina.

En el lenguaje del modelo, esas dinámicas están expresadas por la ecuación (16), donde se muestra, primero, que el cambio estructural es decisivo para fomentar el crecimiento de la producción, de la productividad y a la vez del empleo; y segundo, que una mejora en el patrón de especialización internacional y un avance hacia una participación "de calidad" en el comercio internacional, en términos de sectores, industrias y productos, son necesarios para reducir la informalidad.

El gráfico 5 pone de manifiesto la persistencia, e incluso la acentuación, de las debilidades estructurales de América Latina. En él se muestra el dinamismo de las importaciones respecto del PIB, y la productividad relativa de la región en comparación con la de los Estados Unidos. Si bien es cierto que la importación de tecnologías avanzadas permite modernizar los procesos productivos, ella no genera automáticamente el desarrollo de capacidades tecnológicas endógenas. ${ }^{26}$ De hecho, a contar de mediados del decenio de 1980 la elasticidad-ingreso de las importaciones, que incluyen bienes de capital e insumos de alta tecnología,

\footnotetext{
${ }^{26}$ El incremento de las importaciones de bienes de capital no modificó el patrón de especialización productiva a favor de actividades y sectores con más contenido de conocimiento y más valor agregado. El proceso de apertura propició la reestructuración del sector formal en el sentido de fortalecer la especialización de los países de América Latina en actividades de bajo contenido tecnológico y su participación en cadenas de producción internacionales a niveles jerárquicos subordinados (Cimoli, 2005).
} 


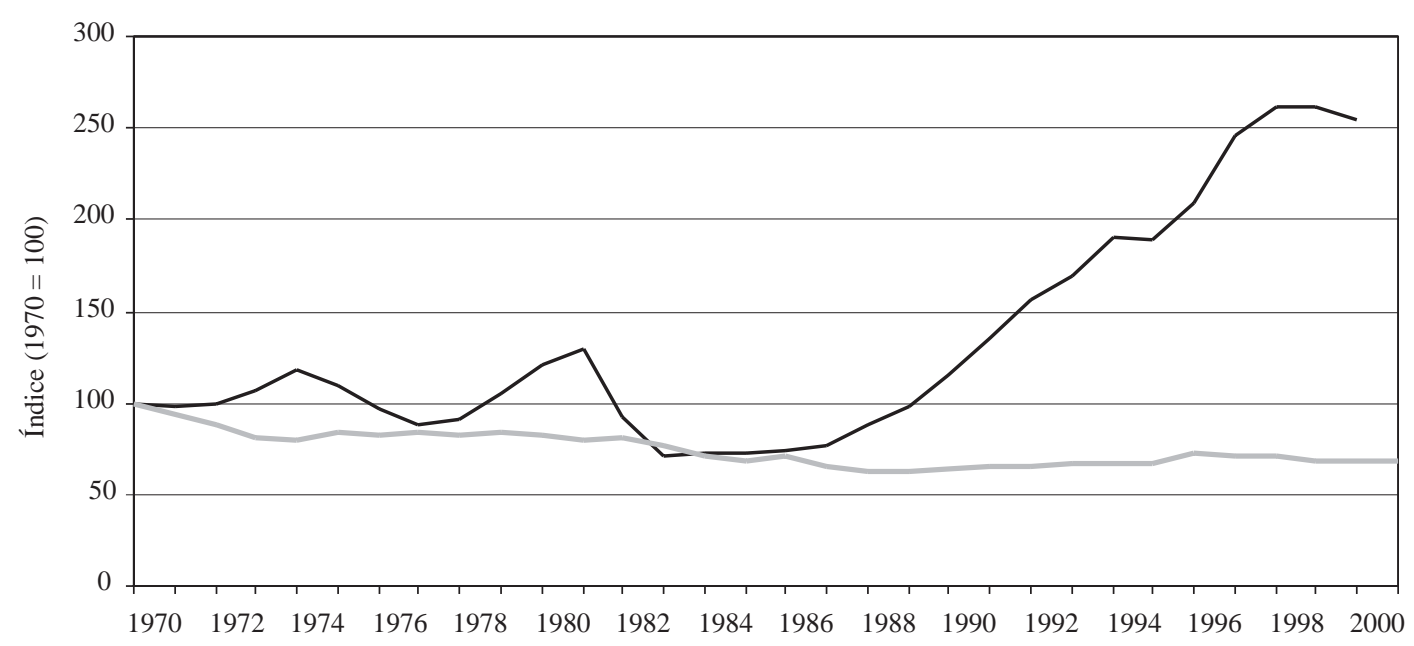

—_ Índice de las importaciones como porcentaje del PIB, América Latina

Índice de la productividad de América Latina en relación con la de Estados Unidos

Fuente: Cimoli, Correa y Primi, 2003.

registró un extraordinario incremento, mientras que la brecha de productividad solo se redujo levemente. ${ }^{27}$

El hecho de que no mejore el patrón de especialización impide que los países latinoamericanos logren mayor competitividad y aumenten las exportaciones, con lo cual empeora la restricción externa y, por ende, se debilita el crecimiento (Cimoli y Correa, 2005; Ocampo y Martin, 2003). En consecuencia, la economía evoluciona en torno a un enclave y ni siquiera asegura una tasa de crecimiento alta. En un contexto caracterizado por el rezago de la estructura productiva respecto de la frontera tecnológica y por una escasa eficiencia en la producción, es posible que la liberalización y el aumento del comercio exterior se traduzcan en una mayor elasticidad-ingreso de las importaciones que contrarreste la dinámica de las exportaciones, como sucedió en América Latina tras la apertura.

${ }^{27}$ En América Latina, el llamado multiplicador del comercio, que corresponde a la relación entre el multiplicador de la brecha tecnológica — esto es, el crecimiento relativo de la productividad en la región respecto de aquel en la frontera tecnológica (de la cual la economía estadounidense es una buena variable sustitutiva) - y la elasticidad ingreso de las importaciones, se redujo de 0,43 a 0,28 después de las reformas, obstaculizando así los efectos positivos del aumento de las exportaciones en el crecimiento del producto (Cimoli, Correa y Primi, 2003).
El modelo puede captar con facilidad los efectos de un incremento de la elasticidad-ingreso de las importaciones. En la ecuación (10), cuando aumenta $\varepsilon$ disminuye $b$. Entonces, como lo ilustra el gráfico 6, la función DR gira en el sentido de los punteros del reloj y disminuyen $y_{f \mathrm{e}}$ y $\pi_{\mathrm{fe}}$. Sin embargo, las ecuaciones (12) y (13) indican que el efecto principal se produce en $y_{f e}$. De hecho, $\frac{\partial \pi_{f e}}{\partial b}=\beta \frac{\partial y_{f e}}{\partial b}$, de manera que desciende el empleo en el sector formal y se eleva en el informal. Como consecuencia, la productividad y la producción agregados disminuyen, ya que en términos relativos se amplía el sector cuyo nivel de productividad es menor y aumenta la brecha de salarios.

El modelo puede también captar los efectos de la pérdida de los encadenamientos y la reducción de la complejidad de las cadenas productivas locales. Una disminución de $\beta$, determina una rotación hacia abajo del régimen de productividad (PR), como en el gráfico 7. En definitiva, se reducen $y_{f \mathrm{e}} \mathrm{y} \pi_{\mathrm{fe}}$. Al elevarse $\alpha$, la función PR se traslada aun más hacia abajo, afectando positivamente $\pi_{f \mathrm{e}} \mathrm{y}$, en menor grado, $y_{f \mathrm{e}}$ (de hecho, $\frac{\partial y_{f e}}{\partial \alpha}=b \frac{\partial \pi_{f e}}{\partial \alpha}$ ). Este cambio de origen del 
GRÁFICO 6

América Latina: Reducción de las tasas de crecimiento de equilibrio de la producción y de la productividad tras el aumento de la elasticidad-ingreso de las importaciones

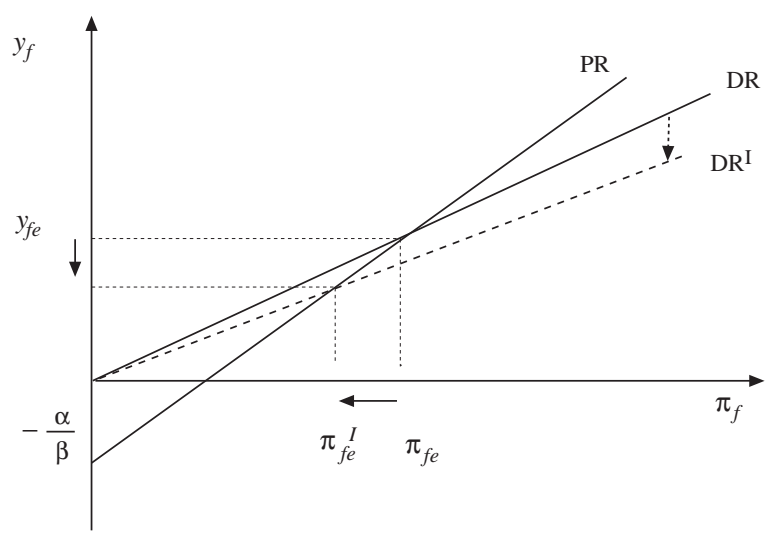

Fuente: Elaboración propia.

crecimiento de la productividad explica que el crecimiento de ella en el sector formal aumente y no disminuya. En otras palabras, es posible que el efecto positivo del aumento de $\alpha$ sobre $y_{\text {fe }}$ contrarreste totalmente los efectos negativos de la disminución de $b$ y $\beta$

\section{$\mathrm{V}$}

\section{Conclusiones}

La heterogeneidad estructural ha sido una barrera de larga data para el crecimiento en América Latina. La convergencia de las economías latinoamericanas hacia aquellas que se encuentran en la frontera tecnológica se ha visto persistentemente dificultada por la coexistencia en la región de un sector formal y otro informal que poseen sistemas productivos e institucionales diferentes.

Las debilidades estructurales y el incremento del comercio internacional han generado incentivos perversos que han favorecido un crecimiento de enclave, en el cual solo el sector formal ${ }^{28}$ se beneficia del mayor comercio mundial y cuyos frutos no se transfieren al "resto" de la economía. La modernización del sector

${ }^{28} \mathrm{Y}$ solo algunos enclaves dentro del sector formal.

\section{GRÁFICO 7}

América Latina: Reducción de las tasas de crecimiento de equilibrio de la producción y de la productividad al disminuir la generación endógena de crecimiento de la productividad

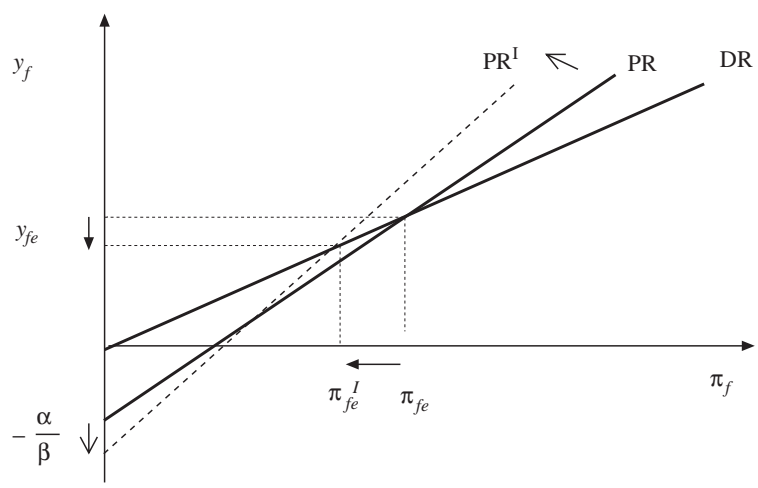

Fuente: Elaboración propia.

sobre $y_{f \mathrm{e}}$. El apéndice B demuestra que en este caso el modelo de todas formas pronostica un aumento de $\pi_{f \mathrm{e}}$ $\mathrm{y}$, por lo tanto, una reducción de $l_{\mathrm{fe}}$, con todas las consecuencias negativas que esto tiene para el crecimiento agregado de la economía.

formal, además, se ha basado en innovaciones de carácter incremental y en la racionalización de los procesos productivos, más que en el aumento de las capacidades tecnológicas endógenas (CEPAL, 2004b); asimismo, los incrementos de productividad se han debido en gran medida a la expulsión de fuerza de trabajo, lo que ha reforzado la persistencia de la informalidad.

Para aliviar las restricciones externas al crecimiento se necesitan estructuras productivas más complejas y diversificadas, con mayor difusión de conocimiento y más encadenamientos, y un cambio en el patrón de especialización hacia productos y procesos que hagan uso más intensivo de conocimiento. En realidad, los esfuerzos por intensificar los encadenamientos hacia adelante y hacia atrás en América Latina solo desembocarán en una disminución del empleo informal si a la vez se transforma la estructura productiva y se 
reorienta el patrón de especialización internacional de los países latinoamericanos.

Para acelerar el ritmo de crecimiento y crecer más, la región tiene que superar su situación actual, orientándose hacia una modalidad de crecimiento "inclusivo" liderado por las exportaciones y acrecentando su

\section{APÉNDICE A}

\author{
La estática del modelo
}

Se considera un tradicional modelo $2 \times 2$ donde $Y,=Y_{f}$, $+Y_{i}$.

La producción de la economía, $Y$, incluye dos tipos de productos: los originados en el sector formal, $Y_{f}$, que utilizan insumos importados y se destinan a la exportación, y los del sector informal, $Y_{i}$, que se destinan totalmente al mercado interno.

El sector formal trabaja básicamente con una tecnología aditiva sencilla que utiliza fuerza de trabajo $\left(L_{f}\right)$ multiplicada por un esfuerzo variable del trabajador $(h)$ e insumos importados $\left(M_{f}\right)^{30}$

$$
Y_{f}=\Pi_{f} L_{f} h+\eta M_{f} \quad \Pi_{f}>0, \eta>0
$$

$\Pi_{f}$ y $\eta$ son los parámetros tecnológicos proporcionados a la empresa, que varían en el largo plazo. En especial, el progreso técnico generalmente aumenta el $\Pi_{f}$, pero posiblemente también requiera sustituir fuerza de trabajo, disminuyendo $\eta$.

La extensión de la ecuación (1) al esfuerzo variable del trabajador es característica del sector formal. Según la teoría de los salarios de eficiencia, el esfuerzo del trabajador será mayor en el sector formal que en el informal. Se supone que el esfuerzo del trabajador en el sector formal es una función positiva (en forma de S) de los salarios, que se ve afectada por las menores remuneraciones del sector informal. La ecuación (2) especifica el esfuerzo del trabajador:

$$
h=\ln q \frac{W_{f}-W_{i}}{\Pi_{f}}
$$

\footnotetext{
${ }^{29}$ En otras palabras, una participación "de calidad" en el comercio internacional es un requisito previo para generar un patrón de crecimiento virtuoso que determine por un lado la caída del empleo informal y por otro el crecimiento de la producción, de la productividad y del empleo en el sector formal.

${ }^{30}$ Para simplificar, no se incluye el uso de insumos domésticos locales en la especificación del sector formal. El supuesto, sin embargo, es coherente con el objetivo del modelo, que apunta a mostrar las diferencias estructurales entre el sector formal y el informal. En realidad, la diferencia principal entre los dos sectores, además de la diferencia de productividad, reside en que el primero se orienta al exterior y el segundo al interior, justificando así lo especificado en la ecuación (1).
}

capacidad de absorber mano de obra. América Latina debería transformar su estructura productiva y reposicionarse en los mercados internacionales, dejando atrás el problema persistente de la informalidad y sentando así las bases para una convergencia de las economías latinoamericanas hacia la frontera tecnológica. ${ }^{29}$

Todas las empresas del sector formal maximizan las utilidades $P f$ controlando los salarios. La existencia de barreras a la entrada permite obtener utilidades positivas. La maximización requiere que $\frac{\partial P_{f}}{\partial W_{f}}=0$, esto es:

$$
\frac{\partial}{\partial W_{f}}\left(Y_{f}-L_{f} W_{f}-p \eta M_{f}\right)=0
$$

en que $p$ es el precio internacional de los insumos importados, en moneda local. De esta manera, la maximización de las utilidades arroja los siguientes resultados después de reemplazar (1) en (3):

$$
\begin{gathered}
W_{f}=\Pi_{f}+W_{i} \\
h=\ln q
\end{gathered}
$$

Sustituyendo (4) en (2) se obtiene la ecuación (5). Esta indica que cuando la productividad aumenta gracias al progreso técnico (y la sustitución de trabajo), y se amplía la brecha salarial entre los dos sectores, el esfuerzo del trabajador no cambia.

Por lo tanto, las empresas pueden establecer un determinado nivel de esfuerzo, que será superior a 1 si $q$ es superior al número neperiano. ${ }^{31}$ Los salarios del sector formal se determinan de acuerdo con la productividad de la mano de obra y a partir del salario del sector informal, que es menor. ${ }^{32}$ De esta manera se conforma un mercado de trabajo segmentado. Como los trabajadores se disputan los empleos formales, las empresas del sector pueden seleccionar el factor trabajo. La demanda dirigida al sector formal determinará a la vez los puestos de trabajo y las importaciones que requerirá este sector, mientras que el sector informal absorberá la oferta residual.

\footnotetext{
31 Cabe destacar que para obtener al menos utilidades positivas, esto es, $P_{f}=L_{f}\left(\Pi_{f}(\ln q-1)-W_{i}\right)+(1-p) \eta M_{f}$, el parámetro $m$ debe ser suficientemente superior al número neperiano.

${ }^{32}$ El tamaño y número de empresas siguen siendo indeterminados.
} 
En el sector informal, la producción se genera con rendimientos constantes del trabajo y con un esfuerzo unitario:

$$
Y_{i}=\Pi_{i} L_{i}
$$

Un supuesto importante para el modelo es que

$$
\Pi_{i}=d \Pi_{f} \quad \text { en que } 0<d<1
$$

APÉNDICE B

$$
\text { El régimen de demanda }
$$

El marco teórico del régimen de demanda (Setterfield y Cornwall, 2002) se remonta al concepto de multiplicador del comercio exterior desarrollado por Harrod (1933), Kaldor (1966 y 1975) y Thirlwall (1979), de acuerdo con el cual la creciente elasticidad-ingreso de las importaciones obstaculiza los efectos positivos que genera el aumento de las exportaciones en el crecimiento del ingreso. En las versiones desarrolladas posteriormente por Cimoli, Dosi y Soete (1986) y Cimoli (1994), al planteamiento original se agregó el concepto de multiplicador de la brecha tecnológica. En la práctica, el aumento del ingreso no solo se ve afectado por el incremento de las exportaciones y la elasticidad-ingreso de las importaciones, sino también por la relación entre la tasa de crecimiento de la productividad del sector formal orientado hacia las exportaciones y la que corresponde a la frontera tecnológica, es decir, el multiplicador de la brecha tecnológica: $\Psi=\frac{\pi_{f}}{\pi^{*}}$.

El régimen de demanda se deriva de una situación de equilibrio de la balanza comercial expresada por la ecuación (i)

$$
M=E
$$

en que $M$ representa la demanda interna de importaciones y $E$ las exportaciones, ambas expresadas en la misma unidad monetaria.

De acuerdo con la teoría clásica de la demanda, la demanda interna de importaciones es una función multiplicadora del ingreso nacional y se determina por la siguiente ecuación:

$$
M=Y_{f}^{\varepsilon}
$$

De la maximización de las utilidades se obtiene que:

$$
W_{i}=\Pi_{I}
$$

Por lo tanto, el sector informal generalmente se caracteriza por la baja productividad. Los salarios se determinan en este sector y consecuentemente en el sector formal mediante la ecuación (4).

en que $\varepsilon$ es la elasticidad-ingreso de las importaciones mientras que $Y_{f}$ representa el ingreso interno generado en el sector formal.

De acuerdo con esto, las exportaciones son una función multiplicadora del ingreso externo $Y^{*}$ y se calculan mediante la ecuación (iii):

$$
E=Y^{* \tau \Psi}
$$

en que $\tau$ representa la elasticidad-ingreso de las exportaciones y $\Psi$ el multiplicador de la brecha tecnológica.

Cuando la ecuación (i) es válida, también lo es su versión dinámica, expresada por la ecuación (iv):

$$
m=e
$$

en que $m$ representa la tasa interna de crecimiento de las importaciones y $e$ la tasa de crecimiento de las exportaciones. Por lo tanto, diferenciando las ecuaciones (ii) y (iii) y reemplazándolas en la ecuación (iv) se obtiene la ecuación (v), que expresa la versión dinámica de la situación de equilibrio de la balanza comercial.

$$
\varepsilon y_{f}=\tau \Psi y^{*}
$$

Suponiendo que $x$ sea el crecimiento de las exportaciones derivado del crecimiento del ingreso externo, $X=\tau \mathrm{y}^{*}$, y sustituyendo la ecuación (iv) en la ecuación (v) y considerando que $b=\frac{x}{\varepsilon \pi *}$ se obtiene la siguiente ecuación multiplicativa, que es igual al régimen de demanda especificado por la ecuación (10): 


\section{APÉNDICE C}

El efecto de un incremento de los esfuerzos autónomos sobre la productividad

Para demostrar que el aumento de $\alpha$ junto con reducciones de $b$ y $\beta$ puede generar un valor constante de $y_{f \mathrm{e}} \mathrm{y}$ un aumento de $\pi_{\mathrm{fe}}$, procedamos en dos etapas. Primero, demostremos que al aumentar simultáneamente $\alpha$ y disminuir $b$ se puede obtener un valor constante de $y_{f \mathrm{e}}$ y un aumento del valor de $\pi_{f \mathrm{e}}$. A partir de la situación de efecto neto cero en $y_{f \mathrm{e}}$, es decir, $\frac{\partial y_{f \mathrm{e}}}{\partial \alpha} \Delta \alpha+\frac{\partial y_{f \mathrm{e}}}{\partial b} \Delta b=0$, obtengamos que $\frac{\Delta b}{\Delta \alpha}=-b \frac{1-b \beta}{\alpha}<0$. A continuación, derivemos los cambios directos e indirectos en $\pi_{f \mathrm{e}}$ para un valor unitario de $\Delta \alpha$, es decir, $\mathrm{d} \pi_{f \mathrm{e}} \frac{\partial \pi_{f \mathrm{e}}}{\partial \alpha}+\frac{\partial \pi_{f \mathrm{e}}}{\partial b} \Delta b=\frac{\partial \pi_{f \mathrm{e}}}{\partial \alpha}-\frac{\partial \pi_{f \mathrm{e}}}{\partial b} b \frac{1-b \beta}{\alpha}>0$. En segundo lugar, demostremos que al aumentar simultáneamente $\alpha$ y disminuir $\beta$ se pueden obtener valores constantes de $y_{f \mathrm{e}}$ y $\pi_{f \mathrm{e}}$. De la misma forma, derivemos $\frac{\Delta \beta}{\Delta \alpha}=-\frac{1-b \beta}{b \alpha}$ y entonces $\mathrm{d} \pi_{f \mathrm{e}}=\frac{\partial \pi_{\mathrm{fe}}}{\partial \alpha}+\frac{\partial \pi_{f \mathrm{e}}}{\partial \beta} \Delta \beta=0$. Por lo tanto, al aumentar $\alpha$ y reducir oportunamente $b$ y $\beta$ se pueden obtener valores constantes de $y_{f \mathrm{e}}$ y un aumento del valor de $\pi_{f \mathrm{e}}$.

\section{Bibliografía}

Agenor, P. y J. Aizenman (1999): Macroeconomic adjustment with segmented labor markets, Journal of Development Economics, vol. $58, \mathrm{~N}^{\mathrm{o}} 2$, Amsterdam, Elsevier.

Amable, B. (1992): Effets d'apprentissage, compétitivité hors prix et croissance cumulative, Économie appliquée, vol. 45, No 3 , París, Institut de Sciences Mathématiques et Economiques Appliquées.

Arbache, J.S. (2001): Wage differentials in Brazil: theory and evidence, Journal of Development Studies, vol. 38, $\mathrm{N}^{\mathrm{o}} 2$, Londres, Taylor \& Francis.

BID (Banco Interamericano de Desarrollo) (2003): Good Jobs Wanted: Labor Markets in Latin America. 2004 Report, Washington, D.C.

Bulow, J.I. y L.H. Summers (1986): A theory of dual labor markets with application to industrial policy, discrimination, and Keynesian unemployment, Journal of Labor Economics, vol. 4, $\mathrm{N}^{\mathrm{o}} 3$, Chicago, The University of Chicago Press.

Carillo, M.R. y M. Pugno (2004): The underground economy and underdevelopment, Economic Systems, vol. 28, $\mathrm{N}^{\mathrm{o}} 3$, Amsterdam, Elsevier.

Castells, M., A. Portes y L. Benton (1989): The Informal Economy: Studies in Advanced Countries and Less Developed Economies, Londres, The Johns Hopkins University Press.

CEPAl (Comisión Económica para América Latina y el Caribe) (2002), Globalización y desarrollo, LC/G.2157(SES.29/3), Santiago de Chile.

(2003): Panorama social de América Latina, 2002-2003, LC/G.2209-P, Santiago de Chile, agosto. Publicación de las Naciones Unidas, $\mathrm{N}^{\circ}$ de venta: S.03.II.G.185.

(2004a): Desarrollo productivo en economías abiertas, LC/G.2234(SES.30/3) Santiago de Chile, junio.

(2004b): Panorama social de América Latina, 2004, LC/L.2220-P, Santiago de Chile. Publicación de las Naciones Unidas, $\mathrm{N}^{\mathrm{o}}$ de venta: S.04.II.G.11.

(2005): Estudio económico de América Latina y el Caribe, 2004-2005, LC/G.2279-P, Santiago de Chile. Publicación de las Naciones Unidas, $\mathrm{N}^{\circ}$ de venta: S.05.II.G.2.

Cimoli, M. (1988): Technological gaps and institutional asymmetries in a North- South model with a continuum of goods, Metroeconomica, vol. 39, № 3, Oxford, Blackwell Publishing. (1994): Look in and specialization (dis)advantages in a structuralist model with endogenous growth, en $\mathrm{J}$. Fagerberg, N. von Tunzelman y B. Verspagen (comps.), The Dynamics of Technology, Trade and Growth, Londres, Edgar Elgar. (comp.) (2005): Heterogeneidad estructural, asimetrías tecnológicas y crecimiento en América Latina, Santiago de Chile, Comisión Económica para América Latina y el Caribe (CEPAL)/Banco Interamericano de Desarrollo (BID).

Cimoli, M. y N. Correa (2005): Trade openness and technological gaps in Latin America: a low growth trap, en J. A. Ocampo (comp.), Beyond Reforms: Structural Dynamics and Macroeconomic Vulnerability, Stanford, Stanford University Press.

Cimoli, M., N. Correa y A. Primi (2003): Crecimiento y estructura productiva en economías abiertas: lecciones de la experiencia de América Latina, Santiago de Chile, Comisión Económica para Améric*a Latina y el Caribe (CEPAL), inédito.

Cimoli, M., G. Dosi y L. Soete (1986): Innovation Diffusion Institutional Differences and Patterns of Trade: a North-South Model, DRC Paper, № 36, Sussex, Reino Unido, Universidad de Sussex.

Cimoli, M., A. Primi y G. Stumpo (2004): Trayectorias productivas y generación de empleo, Santiago de Chile, Comisión Económica para América Latina y el Caribe (CEPAL), inédito.

De Soto, H. (1986): The Other Path: the Invisible Revolution in the Third World, Nueva York, Harper \& Row.

Dosi, G. y C. Freeman (1992): The diversity of development patterns: on the processes of catching up, forging ahead and falling behind, documento presentado en la Conferencia "Economic growth and the structure of long-term development" (Varenna, octubre).

Dosi, G., K. Pavitt y L. Soete (1990): The Economics of Technical Change and International Trade, Hemel Hempstead, Reino Unido, Hervester Wheatsheaf.

Dutt, A. (2001): Income Elasticity of Imports, North-South Trade and Uneven Development, Notre Dame, Indiana, Universidad de Notre Dame, inédito.

Esfahani, H.S. y D. Salehi-Isfahani (1989): Effort observability and worker productivity: towards an explanation of economic dualism, Economic Journal, vol. 99, № 397, Oxford, Blackwell Publishing.

Frenkel, R. y L. Taylor (2006): Real Exchange Rate, Monetary Policy and Employment, DESA Working Paper, $\mathrm{N}^{\circ} 19$, ST/ESA/2006/DWP/19, Nueva York, Naciones Unidas, febrero.

Goldberg, K. y N. Pavcnik (2003): The response of the informal sector to trade liberalization, Journal of Development Economics, vol. 72, № 2, Amsterdam, Elsevier.

Gonzaga, J.F. (2003): Balance-of-payments-constrained economic growth in Brazil, Revista de economía política, vol. 23, $\mathrm{N}^{\mathrm{o}} 1$, São Paulo. 
Harrod, R. (1933): International Economics, Cambridge, Cambridge University Press.

Kaldor, N. (1966): Causes of the Slow Rate of Economic Growth in the United Kingdom, Cambridge, Cambridge University Press. (1975): What is wrong with economic theory?, Quarterly Journal of Economics, vol. 89, $\mathrm{N}^{\circ} 3$, Cambridge, Massachusetts, The MIT Press, agosto.

Kuznets, S. (1980): Recent population trends in less developed countries, and implications for international income inequality, en R. Easterlin (comp.), Population and Economic Change in Developing Countries, Chicago, University of Chicago Press.

Lawson, T.J., G. Palma y J. Sender (1989): Kaldor's contribution to economics: an introduction, Cambridge Journal of Economics, vol. 13, Cambridge, Massachusetts.

Loayaza, N. (1997): The Economics of the Informal Sector: a Simple Model and Some Empirical Evidence from Latin America, Policy Research Working Paper Series, No 1727, Washington, D.C., Banco Mundial.

Maldonado, C. (1995): The informal sector: legalization or laissezfaire?, International Labor Review, vol. 134, No 6, Ginebra, Organización Internacional del Trabajo (OIT).

Maloney, W.F. (2004): Informality revisited, World Development, vol. $32, \mathrm{~N}^{\mathrm{o}} 7$, Amsterdam, Elsevier.

McCombie, J., M. Pugno y B. Soro (comps.) (2002): Productivity Growth and Economic Performance: Essays on Verdoorn's Law, Londres, MacMillan.

McCombie, J. y A.P. Thirlwall (1994): Economic Growth and the Balance of Payments Constraint, Nueva York, St Martin's Press.

Moreno-Brid, J.C. (1999a): Balance of payments constrained growth in Central America: 1950-96, Journal of Post Keynesian Economics, vol. 22, № 1, Nueva York, M.E. Sharpe.

(1999b): Mexico's economic growth and the balance of payments constraint: a cointegration analysis, International Review of Applied Economics, vol. 13, № 2, Londres, Taylor $\&$ Francis.

Nurske, R. (1953): Problems of Capital Formation in Undeveloped Countries, Oxford, Oxford University Press.

Ocampo, J.A. y J. Martin (comps.) (2003): Globalización y desarrollo: una reflexión desde América Latina y el Caribe, Santiago de Chile, Comisión Económica para América Latina y el Caribe (CEPAL)/Alfaomega Colombiana.

OIT (Organización Internacional del Trabajo) (1972): Employment, Incomes and Inequality: A Strategy for Increasing Productive Employment in Kenya, Ginebra.

(2004): Panorama laboral 2004, Lima, Oficina Regional para América Latina y el Caribe, Organización Internacional del Trabajo (OIT).

Paus, E., N. Reinhardt y M. Robinson (2003): Trade liberalization and productivity growth in Latin American manufacturing, 1970-98, Policy Reform, vol. 6, № 1, Londres, Taylor \& Francis.

Perraton, J. (2003): Balance of payments constrained growth in developing countries: an examination of Thirlwall hypothesis, International Review of Applied Economics, vol. 17, $\mathrm{N}^{\circ} 1$, Londres, Taylor \& Francis.

Pinto, A. (1970): Heterogeneidad estructural y modelo de desarrollo reciente de la América Latina, Inflación: raíces estructurales, México, D. F., Fondo de Cultura Económica.

(1976): Naturaleza e implicaciones de la heterogeneidad estructural de la América Latina, El trimestre económico, vol. 37(1), No 145, México, D.F., Fondo de Cultura Económica, enero-marzo.

Piore, M.J. y C.F. Sabel (1984): Mass production as destiny and blind decision, inédito.

Pliego, M. (1997): La evolución de empleo en México, en J. López (comp.), Macroeconomía del empleo, México, D.F., Editorial Porrúa.

Prebisch, R. (1949): El desarrollo económico de la América Latina y algunos de sus principales problemas (E.CN.12/89), Santiago de Chile, Naciones Unidas.

(1962): El desarrollo económico de América Latina y algunos de sus principales problemas, Boletín económico de América Latina, vol. 7, № 1, Santiago de Chile, Comisión Económica para América Latina y el Caribe (CEPAL).

(1970): Transformación y desarrollo: la gran tarea de América Latina, México, D.F., Fondo de Cultura Económica.

Pugno, M. (1998): The stability of Thirlwall's model of economic growth and the balance-of-payments constraint, Journal of Post Keynesian Economics, vol. 20, No 4, Nueva York, M.E. Sharpe.

Ros, J. (2005), El desempleo en América Latina desde 1990, serie Estudios y perspectivas, No 29 , LC/L.2265-P, México, D.F., Sede Subregional de la CEPAL en México. Publicación de las Naciones Unidas, $\mathrm{N}^{\circ}$ de venta: S.05.II.G.9.

Rosenbluth, G. (1994): Informalidad y pobreza en América Latina, Revista de la CEPAL, № 52, LC/G.1824-P, Santiago de Chile, abril.

Sánchez, S., A. Joo y C. Zappala (2001): Constrained global integration, Washington, D.C., Banco Mundial, inédito.

Setterfield, M. y J. Cornwall (2002): A neo-Kaldorian perspective on the rise and decline of the Golden Age, M.A. Setterfield (comp.), The Economics of Demand-led Growth, Cheltenham, Reino Unido, Edward Elgar.

Singer, H.W. (1950): The distribution of gains between investing and borrowing countries, The American Economic Review, vol. $40, \mathrm{~N}^{\circ} 2$, Nashville, Tennessee, American Economic Association, mayo.

Stiglitz, J.A. (1974): Alternative theories of wage determination and unemployment in LDC's: the labor turnover model, Quarterly Journal of Economics, vol. 88, No 2, Cambridge, Massachusetts, The MIT Press.

(1976): The efficiency wage hypothesis, surplus labour, and the distribution of income in LDC's, Oxford Economic Papers, vol. 28, N $\mathrm{N}^{\mathrm{2}}$, Oxford, Reino Unido, Oxford University Press, julio.

Thirlwall, A.P. (1979): The balance of payments constraint as explanation of international growth rate differences, Quarterly Review, Roma, Banca Nazionale del Lavoro, marzo.

(1997): Reflections on the concept of balance of payments constrained growth, Journal of Post Keynesian Economics, vol. 19, $\mathrm{N}^{\mathrm{o}} 3$, Nueva York, M.E. Sharpe.

Tokman, V.E. (1994): Informalidad y pobreza: progreso social y modernización productiva, El trimestre económico, vol. 61(1), $\mathrm{N}^{\circ}$ 241, México, D.F., Fondo de Cultura Económica, eneromarzo.

(1997): Jobs and solidarity: main challenges for the post adjustment in Latin America, L. Emmerij (comp.), Economic and Social Development into the XXI Century, Washington, D.C., Banco Mundial.

(2001): De la informalidad a la modernidad, Santiago de Chile, Organización Internacional del Trabajo (OIT).

Weller, J. (2000): Reformas económicas, crecimiento y empleo: los mercados de trabajo en América Latina y el Caribe, Santiago de Chile, Fondo de Cultura Económica. 